\title{
Re: Postpartum haemorrhage requiring transfusion and risk of cardiovascular disease later in life: a retrospective cohort study
}

Shiho Oide ${ }^{1}$, Qianzhi Wang ${ }^{2}$, Jun Miyata ${ }^{3}$, and Takashi Yoshioka ${ }^{4}$

${ }^{1}$ Yotsuya Medical Cube

${ }^{2}$ Tokyo Metropolitan Children's Medical Center

${ }^{3}$ Osaka University

${ }^{4}$ Fukushima Medical University

March 22, 2021

\section{BJOG exchange}

Title:

Re: Postpartum haemorrhage requiring transfusion and risk of cardiovascular disease later in life: a retrospective cohort study

Shiho Oide, $\mathrm{MD}^{\text {a }}$

Qianzhi Wang, MD ${ }^{\mathrm{b}}$

Jun Miyata, MD ${ }^{\mathrm{c}}$

Takashi Yoshioka, MD, MPH, PhD ${ }^{\mathrm{d}}$

a. Yotsuya Medical Cube, Department of Gynecology, Women's center, Chiyoda, Tokyo 102-0084, Japan. b. Department of General Pediatrics, Tokyo Metropolitan Children's Medical Center, Fuchu, Tokyo 183-8561, Japan. c. Public Health, Department of Social Medicine, Osaka University Graduate School of Medicine, Suita, Osaka 565-0871, Japan. d. Center for Innovative Research for Communities and Clinical Excellence (CiRC ${ }^{2}$ LE), Fukushima Medical University, Fukushima, Fukushima, Japan.

\section{Corresponding author:}

Takashi Yoshioka

Center for Innovative Research for Communities and Clinical Excellence (CiRC ${ }^{2}$ LE), Fukushima Medical University, 1 Hikarigaoka, Fukushima, Fukushima 960-1295, Japan

TEL: +81-24-547-1467

E-mail: yoshioka.takashi.52a@kyoto-u.jp

\section{Running title:}

Importance of validity and describing treatments

Word count:

496/500

Sir, 
We read the article by Cho et al. ${ }^{1}$ with great interest and would like to appreciate the authors for their novel and interesting study on the association between postpartum haemorrhage (PPH) and the occurrence of cardiovascular disease (CVD) beyond the peripartum period. Moreover, the large number of study subjects derived from a comprehensive national database was impressive. However, we wish to point out some issues that may influence the interpretation of their results. With respect to these issues, we have three suggestions for this study.

Our first suggestion is regarding the confounding factors. We believe that there are some important residual and more suitable confounding factors in the present study. First, the regular use of heparin or aspirin around the time of delivery can be important residual confounding factors. This is because they both have risks for haemorrhage and thromboembolism (e.g. , in cases of antiphospholipid syndrome, deep venous thrombosis by any underlying conditions, and high risk of preeclampsia). ${ }^{2,3}$ Second, if possible, adjustment for gestational diabetes mellitus, which is quite common in pregnancy and can be a risk factor for future ischemic heart disease, ${ }^{4}$ may be more suitable than non-gestational diabetes mellitus. Finally, we would recommend adjusting for HELLP syndrome and acute fatty liver of pregnancy as more suitable confounding factors than abnormal liver function test, because these are directly related to bleeding and thrombosis. ${ }^{2}$ We believe that adjustment of such residual and more suitable confounding factors will lead to internally validated results.

Our second suggestion is regarding the external validity of the study. In this study, more than $60 \%$ of the initial population was excluded, resulting in a substantial reduction in external validity. Although the authors have conducted a sensitivity analysis including most of the excluded population, details for this analysis, adjusted confounding factors, description of missing values, and how the authors dealt with them were not available. Such insufficient data presentation will make it difficult for readers to interpret their results regarding external validity. Hence, clarification of the background of the excluded population and detailed methods of sensitivity analysis are important.

Our third suggestion is regarding the presentation of the treatment options for PPH. We can assure the authors that clinicians commonly consider treatments such as hysterectomy and interventional radiology (IVR) for PPH. ${ }^{2}$ These factors could potentially serve as indicators of severity, because severe PPH may be frequently treated with these treatments, and the recipients may have a high risk of developing future CVD. It may be helpful to categorise patients treated with or without hysterectomy or IVR instead of transfusion.

Once again, we thank the authors for providing such novel and relevant study findings. However, as discussed above, we believe that this study may have insufficient internal validity due to the presence of residual and more preferable confounding factors, as well as reduced external validity due to the sample size reduction. We believe that addressing both these issues and a detailed descriptions/analysis regarding hysterectomy and IVR will make future clinical research more informative.

\section{Disclosure of interests:}

We have no conflict of interest to disclose.

\section{Funding:}

None to declare.

\section{Data availability statement:}

This manuscript does not contain any data because of the article type (letter to the editor).

\section{References:}

1. Cho GJ, Lee KM, Kim HY, Han SW, Oh MJ, Chiec L, et al. Postpartum haemorrhage requiring transfusion and risk of cardiovascular disease later in life: a retrospective cohort study. BJOG2021;128:738744. doi: 10.1111/1471-0528.16515. 
2. Prevention and Management of Postpartum Haemorrhage: Green-top Guideline No. 52. BJOG . 2017;124(5):e106-e149. doi: 10.1111/1471-0528.14178.

3. Hastie R, Tong S, Wikström AK, Sandström A, Hesselman S, Bergman L. Aspirin use during pregnancy and the risk of bleeding complications: a Swedish population-based cohort study. Am J Obstet Gynecol2021;224:95.e1-95.e12. doi: 10.1016/j.ajog.2020.07.023.

4. Tobias DK, Stuart JJ, Li S, Chavarro J, Rimm EB, Rich-Edwards J, et al. Association of History of Gestational Diabetes With Long-term Cardiovascular Disease Risk in a Large Prospective Cohort of US Women.JAMA Intern Med 2017;177:1735-1742. doi: 10.1001/jamainternmed.2017.2790. 